\title{
Assessing the structure and meaningfulness of the dissociative subtype of PTSD
}

\author{
Jana Ross ${ }^{1}(1) \cdot$ Gabriel Baník $^{2} \cdot$ Mária Dědová $^{3} \cdot$ Gabriela Mikulášková $^{2} \cdot$ \\ Cherie Armour $^{1}$ (I)
}

Received: 2 May 2017/ Accepted: 22 September 2017/Published online: 29 September 2017

(c) The Author(s) 2017. This article is an open access publication

\begin{abstract}
Purpose Studies conducted in the USA, Canada and Denmark have supported the existence of the dissociative PTSD subtype, characterized primarily by symptoms of depersonalization and derealization. The current study aimed to examine the dissociative PTSD subtype in an Eastern European, predominantly female (83.16\%) sample, using an extended set of dissociative symptoms.

Methods A latent profile analysis was applied to the PTSD and dissociation data from 689 trauma-exposed university students from Slovakia.

Results Four latent profiles of varying PTSD and dissociation symptomatology were uncovered. They were named non-symptomatic, moderate PTSD, high PTSD and dissociative PTSD. The dissociative PTSD profile showed elevations on depersonalization and derealization, but also the alternative dissociative indicators of gaps in awareness and
\end{abstract}

Cherie Armour

c.armour1@ulster.ac.uk

Jana Ross

j.ross@ulster.ac.uk

Gabriel Baník

gabriel.banik@gmail.com

Mária Dědová

maria.dedova@gmail.com

Gabriela Mikulášková

gabriela.mikulaskova@unipo.sk

1 Psychology Research Institute, Ulster University, Coleraine BT52 1SA, Northern Ireland, UK

2 Faculty of Arts, Institute of Psychology, University of Prešov, Prešov, Slovakia

3 Faculty of Arts, School of Psychology, Trnava University in Trnava, Trnava, Slovakia memory, sensory misperceptions and cognitive and behavioural re-experiencing. The core PTSD symptoms of 'memory impairment' and 'reckless or self-destructive behaviour' were also significantly elevated in the dissociative PTSD profile. Moreover, anxiety and anger predicted membership in the dissociative PTSD profile.

Conclusion The results provide support for the proposal that the dissociative PTSD subtype can be characterized by a variety of dissociative symptoms.

Keywords PTSD - Dissociation - Subtype - DSM-5 · Latent profile analysis

\section{Introduction}

The relationship between trauma, PTSD and dissociation has been well documented in the literature $[1,2]$ and a number of different models have been proposed to account for this relationship [3]. Perhaps, the most prominent of these models that has garnered a lot of attention in recent years is the Subtype model of dissociative PTSD. Recognizing the importance of this model, the DSM-5 [4] introduced within its nosology a diagnostic category of a dissociative subtype of PTSD. To qualify for this diagnosis, trauma survivors have to meet the full criteria for PTSD and additionally report experiences of depersonalization and/or derealization, characterized by 'out-of-body' experiences and 'feelings of unreality' respectively. The aim of the current study was to examine the support for the Subtype model of the relationship between PTSD and dissociation.

Dalenberg, Glaser and Alhassoon [5] argued that before a relationship between two variables can be considered a disorder subtype, (1) there needs to be a clear definition of the construct, (2) the disorder and its subtype must have a 
differential structure and/or be fuelled by differential biological mechanisms and (3) the existence of the disorder subtype must be meaningful. With respect to the first criterion, the DSM-5 has defined the dissociative PTSD subtype in terms of depersonalization and derealization symptoms and a number of studies have identified these symptoms in individuals with PTSD [6]. With respect to the differential structure criterion, studies have shown that the dissociative and non-dissociative PTSD do differ in their basic structure (i.e. severity of specific PTSD symptoms). In recent years, statistical techniques of latent class (LCA) and latent profile analysis (LPA) have become popular in comparing the structure of dissociative and nondissociative PTSD. LCA/LPA categorizes individuals into different classes/profiles based on their endorsements or severity of individual symptoms. Since there are currently 20 different PTSD symptoms in DSM-5, which allows for the diagnosis of PTSD to be given in 636,120 different ways [7], LCA and LPA are the ideal methods for investigating the structure of dissociative PTSD.

To date, 11 LCA/LPA studies of dissociative PTSD have been published [6]. These studies were all conducted in the USA, Canada or Denmark and, with the exception of one [8], they all identified one latent class (c.f. [9]) which had high levels of both PTSD and dissociation and additionally one or more latent classes that had different levels of PTSD, but relatively low levels of dissociation. The study by Hansen et al. [8], which did not identify a dissociative class, was conducted with a sample of Danish bank employees, all of whom had been victims of bank robbery. The reason for the absence of a dissociative class in their study could possibly be the clearly defined and specific nature of the traumatic event in their sample (compared to other studies, which examined samples exposed to more severe and/or heterogeneous traumatic experiences), although more research is needed to corroborate this assumption. A few of the existing studies which did identify a dissociative class revealed structural differences in PTSD between the dissociative and non-dissociative classes that had comparable levels of PTSD [10-12]. For example, in a sample of 697 US military veterans, Wolf et al. [13] found that the severity of three out of four DSM-5 PTSD symptom clusters (i.e. re-experiencing, negative alterations in cognitions and mood, arousal) was significantly higher in individuals categorized into the high PTSD with dissociation profile than those categorized into the high PTSD only profile. Studies such as these support the structural requirement for the Subtype model of dissociative PTSD by showing that dissociation can affect the severity of PTSD or the endorsement of individual PTSD symptoms.

Another line of research pioneered by Lanius et al. $[14,15]$ has provided support for the mechanism requirement of the Subtype model of dissociative PTSD. These studies have demonstrated that individuals with dissociative PTSD show differential activation in those brain regions that are implicated in emotion regulation and arousal modulation. More specifically, individuals with PTSD and symptoms of depersonalization or derealization show abnormally high activation in the medial prefrontal cortex and the anterior cingulate cortex and hyper-inhibition of the limbic system [16].

The literature has also supported the meaningfulness requirement for the Subtype model, according to which dissociative and non-dissociative PTSD should differ in their course, effective treatments, co-morbidities or risk factors [5]. Longitudinal studies of PTSD and dissociation have shown that higher baseline levels of dissociation are associated with increased severity of PTSD at a later date $[17,18]$. In terms of differential treatment outcomes, there is some evidence suggesting that dissociation may interfere with treatments for PTSD [19, 20], although not all treatment studies reported such an effect [21, 22]. Substantially more evidence for the meaningfulness requirement comes from research examining the co-morbidities and risk factors for dissociative PTSD. These studies aim to determine if any external variables are differentially related to dissociative and non-dissociative PTSD. Significant results have been reported in relation to different psychopathologies [23, 24], social support and emotional coping style [25], childhood adversities [12, 26] and certain demographic variables [13].

\section{Current study}

The primary aim of the current study was to examine the structure and meaningfulness of the dissociative PTSD subtype. An LPA was applied to data from a trauma-exposed sample of university students from Slovakia, where dissociative PTSD has not been examined yet. Additionally, several variables were examined as potential predictors of the latent profiles. These included gender, anxiety and depression, which have been examined in previous studies [10, 24], but also anger, loneliness and distress tolerance, which have not yet been examined in relation to the dissociative PTSD subtype. Anger is a common sequel of trauma exposure $[27,28]$ and it has been reported to cooccur with both PTSD [29-31] and dissociation [32, 33]. Loneliness has similarly been associated with traumatic exposure [34, 35] and PTSD [36], although to the best of our knowledge it has not yet been examined in relation to dissociation. The final predictor variable examined in this study was distress tolerance, or the ability to withstand negative emotional or other aversive states [37]. Distress tolerance has not been examined in relation to dissociative PTSD before; however, it has been shown to be related to PTSD [38, 39]. 
The current study also examined whether the DSM-5 definition of the dissociative PTSD subtype should be amended to incorporate a wider range of dissociative experiences (in addition to depersonalization and derealization), a possibility previously highlighted by Dorahy and van der Hart [40] and empirically supported by Müllerová et al. in a similar study [10]. To do so, the LPA utilized in the current study was applied to symptoms of PTSD along with four different symptom clusters of dissociation; depersonalization/derealization, sensory misperceptions, gaps in awareness and memory, and cognitive and behavioural re-experiencing [41]. The current study therefore examined an alternative definition of the dissociative PTSD subtype than the one currently adopted by DSM-5.

It was hypothesized that (1) the LPA will uncover several latent profiles, one of which will be characterized by elevated dissociation symptomatology (i.e. dissociative PTSD profile); (2) compared to the other profiles, the dissociative PTSD profile will score higher on all four dissociative symptom clusters; and (3) gender, depression, anxiety, anger, loneliness and distress tolerance will be differentially related to the dissociative PTSD profile relative to the non-dissociative PTSD profiles.

\section{Method}

\section{Participants}

Participants were recruited from six universities in Slovakia through an email inviting them to participate in a large-scale survey about PTSD. The majority of participants completed the survey online, but pen-and-paper versions were also available at two universities. A total of 2032 students accessed the questionnaire. The survey response rate could not be calculated due to some of the participating universities not updating their mailing lists regularly. The effective sample consisted of 689 traumaexposed (see the "Measures" section) participants who completed the relevant measures and had less than $30 \%$ of missing data on any one relevant measure. The average age of participants in the effective sample was 22.69 years $(\mathrm{SD}=5.11)$ and there were 573 females $(83.16 \%)$ and 116 (16.84\%) males.

\section{Measures}

Traumatic experiences were assessed using the Slovak version of the Life Events Checklist for DSM-5 (LEC-5) [42], which enquires about one's exposure to 16 potentially traumatic events and one "other very stressful event or experience". Using a six-point nominal scale (Happened to me, Witnessed it, Learnt about it, Part of my job, Not sure, Does not apply), participants indicated the level of their exposure, with the first four response categories representing a positive endorsement of the experience as per the DSM-5 diagnostic criteria for PTSD [4]. At the end, participants were asked to nominate their worst traumatic event to be used in relation to the assessment of their PTSD symptoms. Of the initial sample of 2032 participants, 1082 completed LEC-5. Participants who did not report any traumatic experiences $(n=28)$, or those who nominated 'other' stressful event as most traumatic ( $n=199)$, were not included in the analysis. In the latter case, it was not possible to determine whether the experience was traumatic enough to qualify as a DSM-5 PTSD trauma. Participants who did not nominate their worst event, but endorsed the 'other' event $(n=31)$, were likewise excluded as their reference event could not be determined.

The Slovak version of the PTSD Checklist for DSM-5 (PCL-5; [43]) was used to assess the 20 DSM-5 symptoms of PTSD. Using a five-point Likert scale ranging from 0 (not at all) to 4 (extremely), participants indicated the extent to which they had been bothered by each symptom in the past month. A cutoff score of 38 was recommended to suggest a probable PTSD diagnosis [44]. Alternatively, following the DSM-5, one or more re-experiencing symptoms, at least one avoidance symptom, two or more negative alterations in cognitions and mood symptoms and at least two arousal symptoms, all rated as $2=$ moderately or higher, can be used to indicate a probable diagnosis. Cronbach's alpha in this study was 0.929 .

Dissociation was assessed using the Dissociative Symptoms Scale (DSS; [41]), which was translated into Slovak language using forward- and back-translation by an experienced translator and a doctoral-level psychologist. The authors of the original scale were consulted during the back-translation process. The DSS is a 20-item measure of four domains of dissociation: depersonalization/derealization, sensory misperceptions, gaps in awareness and memory, and cognitive and behavioural reexperiencing. Using a five-point Likert scale ranging from 0 (not at all) to 4 (more than once a day), participants indicated how much each experience happened to them over the past week. Cronbach's alpha in this study was 0.926 for the whole scale and $0.820,0.838,0.775$ and 0.772 for the subscales of depersonalization/derealization, sensory misperceptions, gaps in awareness and memory, and cognitive and behavioural re-experiencing, respectively.

Anxiety and depression were assessed using their respective subscales from the Slovak version of the Depression, Anxiety and Stress Scale-21 [45]. Participants indicated the degree to which each statement applied to them in the previous week using a four-point Likert scale 
ranging from 0 (did not apply to me at all) to 3 (applied to me very much or most of the time). In this study, Cronbach's alpha was 0.907 for depression and 0.826 for anxiety. The total scores for each subscale were multiplied by 2 as per the guidelines of Lovibond and Lovibond [45].

Anger was assessed with the seven-item version of the Dimensions of Anger Reactions scale [46]. The scale was translated into Slovak language using the procedure outlined above. Participants indicated the degree to which each statement applied to them using a five-point Likert scale ranging from 0 (not at all) to 4 (very much). Cronbach's alpha was 0.848 .

The 20-item Slovak version of the UCLA Loneliness Scale (Version 3; [47]) was used to assess the experiences of loneliness. Participants indicated how often they felt what was described by the statements using a four-point Likert scale ranging from 1 (never) to 4 (always). Cronbach's alpha in this study was 0.928 .

Finally, distress tolerance was assessed using the Slovak version of the Distress Tolerance Scale [48]; a 15-item measure assessing one's ability to withstand negative psychological states. Responses were recorded using a fivepoint Likert scale ranging from 1 (strongly agree) to 5 (strongly disagree). Cronbach's alpha was 0.906.

\section{Analytic plan}

The amount of missing data in the effective sample was $0.096 \%$ and Little's MCAR test suggested that the data was missing completely at random $\left(\chi^{2}=3009.507, d f=2921\right.$, $p=0.124)$. The expectation maximization algorithm in SPSS 23 was used to impute the missing values prior to the analysis, which was conducted in three steps. Firstly, an LPA was used to categorize individuals into latent profiles based on their responses to the 20 PCL-5 items and their mean scores on the four DSS subscales (please note that the DSM-5 PTSD criteria F, G and H were not assessed in the current study). DSS subscale scores were used instead of symptom scores to facilitate model convergence. This data reduction approach was previously used by Wolf et al. [13] and Múllerová et al. [10] and can be especially useful if the number of LPA indicators is large relative to the sample size. Using the 24 PTSD and dissociation indicators (i.e. 20 PCL-5 symptoms and four DSS subscale mean scores), LPA models with increasing numbers of profiles were estimated in Mplus 7.3 [49] using the robust maximum likelihood estimator. The models were compared using Akaike's information criterion (AIC), Bayesian information criterion (BIC) and the sample size-adjusted BIC (SSABIC). Lower relative values of these indices point to a better fitting model. The Lo-Mendell-Rubin-adjusted likelihood ratio test (LMRA) was used for direct comparisons of models with different numbers of profiles. Significant $p$ values $(<0.05)$ indicate that a given model fits better than another with one fewer profile. Entropy was used as an indicator of how clearly delineated the profiles were, with values approaching one indicating an accurate classification of participants into latent profiles.

Once the optimal model was selected, analyses of variance (ANOVAs) were used to compare the PTSD and dissociation indicator scores across the profiles. Finally, the three-step approach for conducting multinomial logistic regressions [50] was used to examine the predictors of latent profile membership. With the exception of gender, all predictor variables (depression, anxiety, anger, loneliness, distress tolerance) were continuous and entered into the model as sum scores.

\section{Results}

\section{Descriptive statistics}

Descriptive statistics are presented in Table 1. Based on the PCL-5 cutoff score of 38, 76 participants (11.03\%) had probable PTSD. Using the DSM-5 diagnostic algorithm, 122 participants $(17.71 \%)$ had probable PTSD. The most frequently nominated worst traumatic events were a transportation accident ( $n=126,18.29 \%)$, natural disaster ( $n=77,11.18 \%)$, physical assault $(n=70,10.16 \%)$, lifethreatening illness or injury $(n=60,8.71 \%)$ and sudden accidental death $(n=59,8.56 \%)$.

\section{Latent profiles}

Table 2 shows the fit statistics of the estimated models. The five-profile model yielded an unreliable solution due to a nonpositive definite first-order derivative product matrix, and will therefore not be discussed here. The values of the AIC, BIC and SSABIC indices were the lowest for the four-profile model, although the LMRA test favoured the two-profile model. All models had good entropy values $(>0.9)$ and therefore the four-profile model was selected as best fitting.

Figure 1 shows the profile plot of the optimal model. Based on the most likely class membership, Profile 1 consisted of $55.30 \%(n=381)$ of the sample and was named non-symptomatic. These individuals scored low on all PTSD and dissociation indicators. Profile 2 consisted of $30.48 \%$ ( $n=210)$ of the sample and was named moderate PTSD. Relative to the non-symptomatic profile, these individuals had elevated PTSD symptomatology and slightly elevated dissociation symptomatology. Profile 3, consisting of $10.74 \%(n=74)$ of the sample, was named high PTSD. These individuals had, on average, higher PTSD symptom scores than those in the non-symptomatic 
Table 1 Descriptive statistics of the full sample and each latent profile

\begin{tabular}{|c|c|c|c|c|c|}
\hline Variable & $\begin{array}{l}\text { Full sample } \\
(N=689)\end{array}$ & $\begin{array}{l}\text { Non-symptomatic } \\
(n=381)\end{array}$ & $\begin{array}{l}\text { Moderate PTSD } \\
(n=210)\end{array}$ & $\begin{array}{l}\text { High PTSD } \\
(n=74)\end{array}$ & $\begin{array}{l}\text { Dissociative PTSD } \\
(n=24)\end{array}$ \\
\hline \multicolumn{6}{|l|}{ Probable PTSD ${ }^{\mathrm{a}}(n)$} \\
\hline Yes & 122 & 0 & 43 & 56 & 23 \\
\hline No & 567 & 381 & 167 & 18 & 1 \\
\hline $\operatorname{PTSD}^{\mathrm{b}}(\mathrm{M} \pm \mathrm{SD})$ & $17.31 \pm 15.01$ & $6.82 \pm 5.01$ & $23.35 \pm 6.44$ & $43.69 \pm 10.08$ & $49.50 \pm 12.26$ \\
\hline Dissociation $^{\mathrm{b}}(\mathrm{M} \pm \mathrm{SD})$ & $7.06 \pm 9.01$ & $3.03 \pm 3.13$ & $9.10 \pm 6.86$ & $11.12 \pm 6.66$ & $40.63 \pm 12.76$ \\
\hline \multicolumn{6}{|l|}{ Gender $(n)$} \\
\hline Male & 116 & 77 & 19 & 14 & 6 \\
\hline Female & 573 & 304 & 191 & 60 & 18 \\
\hline Anxiety $^{\mathrm{b}}(\mathrm{M} \pm \mathrm{SD})$ & $6.80 \pm 7.28$ & $3.60 \pm 4.08$ & $8.51 \pm 6.62$ & $13.30 \pm 9.13$ & $22.50 \pm 7.67$ \\
\hline Depression $^{\mathrm{b}}(\mathrm{M} \pm \mathrm{SD})$ & $7.64 \pm 9.08$ & $3.80 \pm 4.72$ & $8.72 \pm 8.13$ & $17.35 \pm 10.51$ & $29.33 \pm 9.79$ \\
\hline $\operatorname{Anger}^{\mathrm{b}}(\mathrm{M} \pm \mathrm{SD})$ & $6.96 \pm 5.64$ & $5.15 \pm 4.21$ & $7.66 \pm 5.06$ & $10.88 \pm 7.38$ & $17.33 \pm 5.70$ \\
\hline Loneliness $^{\mathrm{b}}(\mathrm{M} \pm \mathrm{SD})$ & $40.05 \pm 10.90$ & $36.18 \pm 8.81$ & $42.23 \pm 10.17$ & $48.43 \pm 11.60$ & $56.58 \pm 10.06$ \\
\hline $\begin{array}{l}\text { Distress tolerance }^{\mathrm{b}} \\
(\mathrm{M} \pm \mathrm{SD})\end{array}$ & $51.10 \pm 11.90$ & $54.81 \pm 11.23$ & $48.46 \pm 10.21$ & $43.49 \pm 12.33$ & $38.88 \pm 9.57$ \\
\hline
\end{tabular}

${ }^{a}$ Probable PTSD based on the DSM-5 diagnostic algorithm

b Total score

Table 2 Fit indices of the estimated latent profile models

Fig. 1 Four-class profile plot of the PTSD symptom scores and the dissociation subscale scores

\begin{tabular}{llllll}
\hline Model & AIC & BIC & SSABIC & LMRA $p$ value & Entropy \\
\hline One profile & $46,761.022$ & $46,978.714$ & $46,826.306$ & - & - \\
Two profiles & $41,941.364$ & $42,272.436$ & $42,040.650$ & 0.0001 & 0.971 \\
Three profiles & $40,514.021$ & $40,958.475$ & $40,647.310$ & 0.3071 & 0.961 \\
Four profiles & $39,647.783$ & $40,205.618$ & $39,815.074$ & 0.2246 & 0.940 \\
\hline
\end{tabular}

AIC Akaike's information criterion, BIC Bayesian information criterion, LMRA Lo-Mendell-Rubin-adjusted likelihood ratio test, SSABIC sample size-adjusted BIC

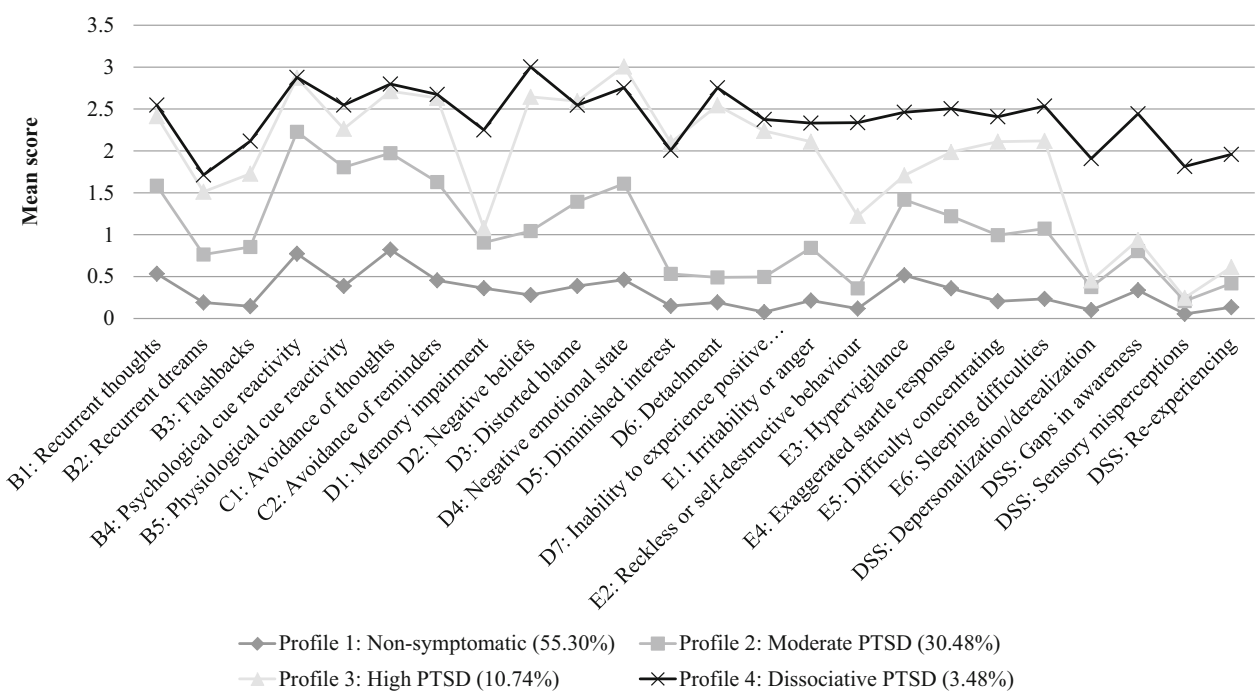

and moderate PTSD profiles, but their scores on dissociation indicators remained relatively low. Finally, Profile 4 was the smallest subgroup, consisting of $3.48 \%(n=24)$ of the sample. With the exception of a few PTSD symptoms, it was characterized by PTSD symptomatology comparable to that in the high PTSD profile, but it had elevated scores on all four dissociation indicators. Profile 4 was therefore named dissociative PTSD. 
Table 3 Odds ratios and 95\% confidence intervals for predictors of the latent profiles

\begin{tabular}{|c|c|c|c|c|c|c|}
\hline Predictors & $\begin{array}{l}\text { Non-symptomatic }{ }^{\mathrm{a}} \\
\text { vs. moderate PTSD }\end{array}$ & $\begin{array}{l}\text { Non-symptomatic }{ }^{a} \\
\text { vs. high PTSD }\end{array}$ & $\begin{array}{l}\text { Non-symptomatic } \\
\text { vs. dissociative PTSD }\end{array}$ & $\begin{array}{l}\text { Moderate PTSD } \\
\text { vs. high PTSD }\end{array}$ & $\begin{array}{l}\text { Moderate PTSD } \\
\text { vs. dissociative } \\
\text { PTSD }\end{array}$ & $\begin{array}{l}\text { High PTSD }^{\mathrm{a}} \\
\text { vs. dissociative } \\
\text { PTSD }\end{array}$ \\
\hline Male sex ${ }^{b}$ & $\begin{array}{l}0.324 \\
\quad(0.162-0.650)^{* *}\end{array}$ & $\begin{array}{l}0.761 \\
\quad(0.319-1.814)\end{array}$ & $\begin{array}{l}1.826 \\
\quad(0.478-6.977)\end{array}$ & $\begin{array}{l}2.347 \\
\quad(1.004-5.483)^{*}\end{array}$ & $\begin{array}{l}5.629 \\
\quad(1.526-20.767)^{* *}\end{array}$ & $\begin{array}{l}2.399 \\
(0.695-8.279)\end{array}$ \\
\hline Anxiety & $\begin{array}{l}1.125 \\
\quad(1.069-1.184)^{* * *}\end{array}$ & $\begin{array}{l}1.138 \\
\quad(1.069-1.211)^{* * *}\end{array}$ & $\begin{array}{l}1.280 \\
(1.152-1.423)^{* * *}\end{array}$ & $\begin{array}{l}1.011 \\
\quad(0.963-1.062)\end{array}$ & $\begin{array}{l}1.138 \\
\quad(1.034-1.252)^{* *}\end{array}$ & $\begin{array}{l}1.125 \\
\quad(1.022-1.239)^{*}\end{array}$ \\
\hline Depression & $\begin{array}{l}1.062 \\
(1.013-1.113)^{* *}\end{array}$ & $\begin{array}{l}1.135 \\
\quad(1.073-1.202)^{* * *}\end{array}$ & $\begin{array}{l}1.195 \\
\quad(1.094-1.305)^{* * * *}\end{array}$ & $\begin{array}{l}1.069 \\
(1.026-1.114)^{* *}\end{array}$ & $\begin{array}{l}1.125 \\
(1.038-1.219)^{* *}\end{array}$ & $\begin{array}{l}1.052 \\
(0.975-1.136)\end{array}$ \\
\hline Anger & $\begin{array}{l}1.024 \\
\quad(0.975-1.076)\end{array}$ & $\begin{array}{l}1.060 \\
(0.986-1.139)\end{array}$ & $\begin{array}{l}1.206 \\
(1.080-1.345)^{* *}\end{array}$ & $\begin{array}{l}1.035 \\
(0.974-1.099)\end{array}$ & $\begin{array}{l}1.177 \\
\quad(1.063-1.303)^{* *}\end{array}$ & $\begin{array}{l}1.139 \\
\quad(1.035-1.254)^{* *}\end{array}$ \\
\hline Loneliness & $\begin{array}{l}1.038 \\
(1.014-1.062)^{* *}\end{array}$ & $\begin{array}{l}1.047 \\
(1.007-1.089)^{*}\end{array}$ & $\begin{array}{l}1.036 \\
(0.967-1.109)\end{array}$ & $\begin{array}{l}1.008 \\
(0.973-1.044)\end{array}$ & $\begin{array}{l}0.998 \\
\quad(0.932-1.069)\end{array}$ & $\begin{array}{l}0.990 \\
\quad(0.928-1.056)\end{array}$ \\
\hline $\begin{array}{l}\text { Distress } \\
\text { tol. }\end{array}$ & $\begin{array}{l}0.983 \\
\quad(0.962-1.005)\end{array}$ & $\begin{array}{l}0.969 \\
\quad(0.937-1.001)\end{array}$ & $\begin{array}{l}1.001 \\
\quad(0.942-1.064)\end{array}$ & $\begin{array}{l}0.985 \\
\quad(0.957-1.015)\end{array}$ & $\begin{array}{l}1.018 \\
(0.960-1.080)\end{array}$ & $\begin{array}{l}1.034 \\
(0.976-1.094)\end{array}$ \\
\hline
\end{tabular}

$*<.05 ; * *<.01 ; * * *<.001$

${ }^{\text {a }}$ Reference profile

b 'Female' was the reference category

\section{PTSD and dissociation as a function of latent profile membership}

The ANOVAs examining the differences in PTSD and dissociation symptomatology as a function of latent profile membership were all significant $\left(p_{\mathrm{s}}<0.001\right)$, even after applying the Bonferroni correction to account for multiple comparisons. The Games-Howell post hoc tests, which are suitable for examining differences between samples of unequal sizes and with unequal variances, showed that all PTSD and dissociation scores in the non-symptomatic profile were significantly lower $\left(p_{\mathrm{s}}<0.001\right)$ than those in the other three profiles. Cohen's $d$ effect sizes for PTSD ranged from 0.440 to 2.909 and for dissociation from 0.721 to 3.082. When comparing the moderate PTSD with the high PTSD profile, the latter one had significantly higher scores on all PTSD symptoms ( $p_{\mathrm{s}} \leq 0.001$, Cohen's $d=0.534$ to 2.297 ) except for B5, D1 and E3 (see Fig. 1 for symptom descriptions). The high PTSD profile also had significantly higher scores on the dissociative subscale of cognitive and behavioural re-experiencing ( $p=0.033$, Cohen's $d=0.398$ ), but none of the other dissociative subscales. Comparing the moderate PTSD profile with the dissociative PTSD profile, the latter scored significantly higher on all PTSD symptoms $\left(p_{\mathrm{s}}<0.05\right.$, Cohen's $d=0.637$ to 1.990 ), except for B4 and B5, and it also scored higher on all dissociation indicators $\left(p_{\mathrm{s}}<0.001\right.$, Cohen's $d=2.017$ to 2.687 ). Finally, the high PTSD and the dissociative PTSD profiles did not differ significantly on any PTSD symptoms, except for D1 ( $p=0.005$, Cohen's $d=0.866$ ) and E2 ( $p=0.008$, Cohen's $d=0.821)$, where the dissociative PTSD profile scored significantly higher. The dissociative PTSD profile also scored significantly higher than the high PTSD profile on all four dissociation indicators $\left(p_{\mathrm{s}}<0.001\right)$. The effect sizes ranged from 1.765 for cognitive and behavioural re-experiencing to 2.623 for Sensory misperceptions.

\section{Predictors of latent profiles}

The results of the multinomial logistic regressions are presented in Table 3. Males were significantly less likely to be in the moderate PTSD profile than in the non-symptomatic, high PTSD or dissociative PTSD profiles. There was no differentiation based on gender between the high PTSD and dissociative PTSD profiles. In relation to anxiety, for every one point increase on the anxiety measure, individuals were significantly more likely to be in the moderate PTSD, the high PTSD or the dissociative PTSD profiles than the non-symptomatic profile, and significantly more likely to be in the dissociative PTSD profile than the moderate or high PTSD profiles. Depression emerged as a significant predictor of the non-dissociative PTSD profiles, such that for every one point increase in depression, individuals were significantly more likely to be in the higher severity PTSD profiles. There was no differentiation based on depression between the high PTSD and the dissociative PTSD profiles. Anger was a significant predictor of the dissociative PTSD profile relative to all other profiles, such that higher levels of anger predicted membership in the dissociative PTSD profile. Interestingly, there was no differentiation based on anger between the non-dissociative PTSD profiles. Loneliness was a significant predictor of the moderate PTSD and high PTSD profiles relative to the nonsymptomatic profile and distress tolerance did not predict membership in any of the latent profiles. 


\section{Discussion}

The current study examined the structure and meaningfulness of the dissociative PTSD subtype, using an extended set of dissociative symptoms. The results of the LPA revealed four latent profiles differing in the severity of PTSD and dissociation symptomatology: non-symptomatic, moderate PTSD, high PTSD and dissociative PTSD. The last two profiles differed primarily in the severity of dissociation indicators, which was higher in the dissociative PTSD profile, thus supporting hypothesis 1 . Additionally, the dissociative PTSD profile had elevated scores on all dissociation indicators (not just depersonalization and derealization), which supports hypothesis 2 . Finally, hypothesis 3 was partly supported, as anxiety and anger, but none of the other variables, differentially predicted membership in the dissociative PTSD profile relative to the high PTSD profile.

In line with previous studies [9-13, 23-26, 51], the current results support the existence of the dissociative PTSD subtype, as only a small proportion of individuals with elevated PTSD symptomatology also reported elevated dissociative symptoms. The results also contribute to the notion that dissociative PTSD is not a culture-specific construct limited to the USA, Canada and Denmark, as the current study identified its existence in a sample from Slovakia, where this construct had not been examined before. Stein et al. [52] (non-LCA/LPA study) examined the existence of the dissociative subtype in the World Mental Health Survey conducted across 16 countries and found that $14.4 \%$ of individuals with a 12 -month diagnosis of PTSD reported symptoms of dissociation. The current study adds to the findings of Stein et al. who did not include Slovakia in their investigation, although they did include two Eastern European countries; Bulgaria and Romania.

The results further showed that the high PTSD and the dissociative PTSD profiles differed significantly not only in the severity of dissociative symptoms, but also in the severity of two core PTSD symptoms; memory impairment (D1) and reckless or self-destructive behaviour (E2). These findings support the structural requirement for the subtype hypothesis of dissociative PTSD [5], because dissociation in the current sample essentially changed the structure of PTSD. The different severity of the memory impairment symptom in the two latent profiles is not surprising. Indeed, it has previously been suggested that psychogenic amnesia is a symptom of dissociation [40, 53, 54]. Stein et al. [52] reported that in their sample of 25,018 respondents, amnesia and flashbacks were the only PTSD symptoms that were significantly associated with depersonalization and derealization. Interestingly, there was no differentiation based on flashbacks between the two profiles in the current study.

In relation to symptom E2 (reckless or self-destructive behaviour), which was only recently added to the DSM PTSD criteria [4], Frewen et al. [9] found that individuals in their severe dissociative PTSD profile, as well as those in the moderate dissociative PTSD profile endorsed this symptom with greater severity than individuals in the severe non-dissociative PTSD profile. Taken together, the findings of the current study and those of Frewen et al. seem to suggest that the PTSD symptom E2 could be specific to the dissociative PTSD subtype.

Further support for memory impairment and reckless or self-destructive behaviour symptoms as poor indicators of (non-dissociative) PTSD comes from the PTSD confirmatory factor analytic literature, where substantially low factor loadings have been reported for the two symptoms [55-57]. Memory impairment and reckless or self-destructive behaviour symptoms may therefore potentially be better indicators of the dissociative PTSD subtype than the non-dissociative PTSD.

The current study also demonstrated that the dissociative PTSD profile could be identified on the basis of an extended set of dissociative symptoms. The DSS subscales of depersonalization/derealization, sensory misperceptions, gaps in awareness and memory, and cognitive and behavioural re-experiencing all differentiated between the high PTSD and the dissociative PTSD profiles. The DSM-5 limits the diagnosis of dissociative PTSD to symptoms of depersonalization and derealization; however, the current study showed that such a restriction may be inaccurate. This conclusion is supported by the existing literature, according to which a wide range of dissociative symptoms may follow traumatic exposure [58]. So far, there have only been two LPA studies that examined an extended set of dissociative symptoms in PTSD [10, 13]. The study by Múllerová et al. [10] used the same measures of PTSD and dissociation as the current study and it similarly found that the dissociative PTSD profile could be characterized by elevated scores on all DSS subscales, with Cohen's $d$ effect size being largest for the subscale of cognitive and behavioural re-experiencing. In the current study, the largest effect size was found for sensory misperceptions. These findings will need further replications; however, both studies suggest that the symptoms of depersonalization and derealization should not be given precedence in the diagnosis of the dissociative PTSD subtype.

The current study also examined the meaningfulness requirement for the subtype hypothesis of dissociative PTSD by looking at variables that could potentially predict membership in the dissociative PTSD profile relative to the high PTSD profile. Contrary to our predictions, there was no difference based on depression, gender, loneliness and 
distress tolerance between the two profiles. To date, loneliness and distress tolerance have not been examined as predictors of dissociative PTSD and the results regarding depression and gender have been mixed so far [10, 24, 26]. Further research employing these variables is needed to establish their predictive value for dissociative PTSD. Anxiety and anger have, however, emerged as significant predictors of the dissociative PTSD profile in the current study. Specifically, individuals with high levels of PTSD who reported high levels of anxiety were more likely to be in the dissociative PTSD profile than the high PTSD profile. Such results have been reported in several of the previous studies [10, 23, 24], suggesting that anxiety may be an important risk factor for dissociative PTSD. Considering the fact that the high and dissociative PTSD profiles in the current sample had comparable levels of PTSD, it can be suggested that anxiety contributes to the expression of dissociative symptoms in the dissociative PTSD subtype, above and beyond its relationship with PTSD. This finding supports the previously suggested notion that dissociative PTSD is associated with greater co-morbidity [26]. It is also in line with the proposition that dissociation can serve as a defence mechanism [59] against adverse emotional states, such as anxiety. However, considering the fact that the relationship between anxiety and the dissociative PTSD subtype in previous studies has not been consistent [6], further research is needed to disentangle this relationship.

Our results also showed that higher levels of anger were significantly associated with the dissociative PTSD profile membership relative to all non-dissociative PTSD profiles. However, there was no differentiation based on anger between any of the non-dissociative profiles. These findings provide support for the specificity of anger for the dissociative PTSD subtype and concur with previous studies which have found a significant association between anger and dissociation even after controlling for PTSD severity [32, 33]. Feeny et al. [32] argued that anger and dissociation are related, because they are complementary forms of emotional disengagement from traumatic memories. Both methods prevent the processing of traumatic memories, thus hindering recovery. More research is, however, needed to better understand why anger would be a differential predictor of dissociative PTSD relative to non-dissociative PTSD.

\section{Limitations}

Certain limitations of the current study need to be acknowledged. First, the DSS items were not queried in the context of participants' worst trauma, which could potentially mean that they did not result from the traumatic event. Second, the indicators for the LPA were individual
PTSD symptoms, whereas for dissociation we used mean subscale scores. This prevented us from determining the importance of the individual dissociative symptoms for the dissociative PTSD subtype. Nevertheless, considering the relatively small sample size, this approach enabled us to use a full validated measure of dissociation. Third, all data was collected using self-report measures rather than the gold-standard clinical interviews. In relation to the PCL-5, this measure can only be used to indicate a probable PTSD diagnosis. However, in previous studies [60, 61], it showed good psychometric properties and in the current study it had good internal consistency. Nevertheless, the study will need to be replicated using structured clinical interviews. Future studies could utilize samples consisting of only PTSD-diagnosed individuals to see if similar latent profiles emerge. Finally, the use of a predominantly female nonclinical student sample limits the generalizations to male and/or clinical samples. However, having said that, two of the previous LPA studies replicated their findings in a subsample of their participants who met the PTSD criteria $[12,24]$, thus suggesting some degree of generalizability to clinical samples. The effects of gender on dissociative PTSD are not yet fully understood and therefore generalizations from this study should only be made with caution, as $83.16 \%$ of participants were female.

\section{Implications}

Despite these limitations, the results of this study have important implications. Firstly, since all of the DSS subscale scores were significantly elevated in the dissociative PTSD profile, limiting the dissociative PTSD subtype to symptoms of depersonalization and derealization may be inaccurate. Some individuals may display dissociative symptoms other than depersonalization and derealization and experience similar functional impairment or treatment non-response as those with depersonalization and derealization. Further research in this area is needed, but if the results are replicated in future studies, a revision to the DSM-5 diagnostic criteria for PTSD may be necessary. Secondly, the PTSD symptoms D1 and E2 appear to be better indicators of the dissociative PTSD subtype than the non-dissociative PTSD, as they were more highly endorsed by individuals in the dissociative PTSD profile than all others. If replicated in future studies, the designation of these symptoms as core symptoms of PTSD may need to be reconsidered. Thirdly, anger was found to be a specific predictor of dissociative PTSD, which may have implications for treatments of individuals with the dissociative PTSD subtype, as there is some evidence suggesting that anger may interfere with exposure treatments for PTSD [62-64]. There is also some evidence suggesting that dissociation may interfere with treatments $[19,20]$. Future 
studies should therefore examine the effects of different types of treatments on dissociative PTSD as well as different techniques to counteract dissociative responding in therapy [65], both in individuals who do and those who do not display anger. Finally, the identification of a dissociative subtype in a European sample suggests that the omission of this diagnostic construct from the proposed 11th edition of the International Classification of Diseases may be a mistake.

\section{Conclusion}

The current study used LPA to investigate the Subtype hypothesis of dissociative PTSD in a sample of university students from Slovakia. Strong support was found for both the structural and meaningfulness requirement of the Subtype hypothesis. In terms of the definitional requirement, the results suggest that the set of dissociative symptoms characterizing the DSM-5 dissociative PTSD subtype could be extended to include alternative dissociative experiences. Further studies with different populations and examining a variety of dissociative symptoms are warranted.

\section{Compliance with ethical standards}

Ethical standards The study was approved by the Ulster University Psychology filter ethics committee and was performed in accordance with the ethical standards laid down in the 1964 Declaration of Helsinki and its later amendments.

Informed consent Informed consent was obtained from all participants prior to their inclusion in the study.

Conflict of interest The authors declare that they have no conflict of interest.

Open Access This article is distributed under the terms of the Creative Commons Attribution 4.0 International License (http://crea tivecommons.org/licenses/by/4.0/), which permits unrestricted use, distribution, and reproduction in any medium, provided you give appropriate credit to the original author(s) and the source, provide a link to the Creative Commons license, and indicate if changes were made.

\section{References}

1. Gershuny BS, Thayer JF (1999) Relations among psychological trauma, dissociative phenomena, and trauma-related distress: a review and integration. Clin Psychol Rev 19(5):631-657

2. Kessler RC, Sonnega A, Bromet E et al (1995) Posttraumatic stress disorder in the National Comorbidity Survey. Arch Gen Psychiatry 52:1048-1060

3. Dalenberg CJ, Carlson EB (2012) Dissociation in posttraumatic stress disorder part II: how theoretical models fit the empirical evidence and recommendations for modifying the diagnostic criteria for PTSD. Psychol Trauma Theory Res Pract Policy 4(6):551-559. doi:10.1037/a0027900

4. American Psychiatric Association (2013) Diagnostic and statistical manual of mental disorders, 5th edn. Author, Washington DC

5. Dalenberg CJ, Glaser D, Alhasson OM (2012) Statistical support for subtypes in posttraumatic stress disorder: the how and why of subtype analysis. Depression Anxiety 29:671-678. doi:10.1002/ da. 21926

6. Hansen M, Ross J, Armour C (2017) Evidence of the dissociative PTSD subtype: a systematic literature review of latent class and profile analytic studies of PTSD. J Affect Disord. doi:10.1016/j. jad.2017.02.004 (Advance online publication)

7. Galatzer-Levy IR, Bryant RA (2013) 636,120 ways to have posttraumatic stress disorder. Perspect Psychol Sci 8(6):651-662. doi: $10.1177 / 1745691613504115$

8. Hansen M, Hyland P, Armour C (2016) Does highly symptomatic class membership in the acute phase predict highly symptomatic classification in victims 6 months after traumatic exposure? J Anxiety Disord 40:44-51. doi:10.1016/j.janxdis.2016.04.008

9. Frewen PA, Brown MFD, Steuwe C et al (2015) Latent profile analysis and principal axis factoring of the DSM-5 dissociative subtype. Eur J Psychotraumatol 6:26406. doi:10.3402/ejpt.v6. 26406

10. Múllerová J, Hansen M, Contractor AA et al (2016) Dissociative features in posttraumatic stress disorder: a latent profile analysis. Psychol Trauma Theory Res Pract Policy 8(5):601-608. doi:10. 1037/tra0000148

11. Wolf EJ, Lunney CA, Miller MW et al (2012) The dissociative subtype of PTSD: a replication and extension. Depression Anxiety 29:679-688. doi:10.1002/da.21946

12. Wolf EJ, Miller MW, Reardon AF et al (2012) A latent class analysis of dissociation and posttraumatic stress disorder: evidence for a dissociative subtype. Arch Gen Psychiatry 69(7):698-705. doi:10.1001/archgenpsychiatry.2011.1574

13. Wolf EJ, Mitchell KS, Sadeh N et al (2015) The Dissociative Subtype of PTSD Scale: initial evaluation in a national sample of trauma-exposed veterans. Assess. doi:10.1177/ 1073191115615212

14. Lanius RA, Williamson PC, Boksman K et al (2002) Brain activation during script-driven imagery induced dissociative responses in PTSD: a functional magnetic resonance imaging investigation. Biol Psychiatry 52:305-311

15. Lanius RA, Williamson PC, Densmore M et al (2001) Neural correlates of traumatic memories in posttraumatic stress disorder: a functional MRI investigation. Am J Psychiatry 158:1920-1922. doi:10.1176/appi.ajp.158.11.1920

16. Lanius RA, Brand B, Vermetten E et al (2010) Emotion modulation in PTSD: clinical and neurobiological evidence for a dissociative subtype. Am J Psychiatry 167(6):640-647. doi:10.1176/ appi.ajp.2009.09081168

17. Murray J, Ehlers A, Mayou RA (2002) Dissociation and posttraumatic stress disorder: two prospective studies of road traffic accident survivors. Br J Psychiatry 180:363-368. doi:10.1192/ bjp. 180.4 .363

18. Werner KB, Griffin MG (2012) Peritraumatic and persistent dissociation as predictors of PTSD symptoms in a female cohort. J Trauma Dissociation 25(4):401-407. doi:10.1002/jts.21725

19. Bae H, Kim D, Park YC (2016) Dissociation predicts treatment response in eye-movement desensitization and reprocessing for posttraumatic stress disorder. J Trauma Dissociation 17(1):112-130. doi:10.1080/15299732.2015.1037039

20. Price M, Kearns M, Houry D et al (2014) Emergency department predictors of posttraumatic stress reduction for trauma-exposed individuals with and without an early intervention. J Consult Clin Psychol 82(2):336-341. doi:10.1037/a0035537 
21. Hagenaars MA, van Minnen A, Hoogduin KAL (2010) The impact of dissociation and depression on the efficacy of prolonged exposure treatment for PTSD. Behav Res Ther 48:19-27. doi:10.1016/j.brat.2009.09.001

22. Halvorsen JØ, Stenmark H, Neuner F et al (2014) Does dissociation moderate treatment outcomes of narrative exposure therapy for PTSD? A secondary analysis from a randomized controlled clinical trial. Behav Res Ther 57:21-28. doi:10.1016/j. brat.2014.03.010

23. Armour C, Elklit A, Lauterbach D et al (2014) The DSM-5 dissociative-PTSD subtype: can levels of depression, anxiety, hostility, and sleeping difficulties differentiate between dissociativePTSD and PTSD in rape and sexual assault victims? J Anxiety Disord 28:418-426. doi:10.1016/j.janxdis.2013.12.008

24. Blevins CA, Weathers FW, Witte TK (2014) Dissociation and posttraumatic stress disorder: a latent profile analysis. J Trauma Stress 27(4):388-396. doi:10.1002/jts.21933

25. Hansen M, Müllerová J, Elklit A et al (2016) Can the dissociative PTSD subtype be identified across two distinct trauma samples meeting caseness for PTSD? Soc Psychiatry Psychiatr Epidemiol 51(8):1159-1169. doi:10.1007/s00127-016-1235-2

26. Steuwe C, Lanius RA, Frewen PA (2012) Evidence for a dissociative subtype of PTSD by latent profile and confirmatory factor analyses in a civilian sample. Depression Anxiety 29:689-700. doi:10.1002/da.21944

27. Blaszczynski A, Gordon K, Silove D et al (1998) Psychiatric morbidity following motor vehicle accidents: a review of methodological issues. Compr Psychiatry 39(3):111-121

28. Keene AC, Epps J (2016) Childhood physical abuse and aggression: shame and narcissistic vulnerability. Child Abuse Negl 51:276-283. doi:10.1016/j.chiabu.2015.09.012

29. Asmundson GJG, LeBouthillier DM, Parkerson HA et al (2016) Trauma-exposed community-dwelling women and men respond similarly to the DAR-5 Anger Scale: factor structure invariance and differential item functioning. J Trauma Stress 29:214-220. doi: $10.1002 /$ jts. 22098

30. Jakupcak M, Conybeare D, Phelps L et al (2007) Anger, hostility, and aggression among Iraq and Afghanistan war veterans reporting PTSD and subthreshold PTSD. J Trauma Stress 20(6):945-954. doi:10.1002/jts.20258

31. Worthen M, Rathod SD, Cohen G et al (2016) Anger and posttraumatic stress disorder symptom severity in a trauma-exposed military population: differences by trauma context and gender. J Trauma Stress 29:1-8. doi:10.1002/jts.22050

32. Feeny NC, Zoellner LA, Foa EB (2000) Anger, dissociation, and posttraumatic stress disorder among female assault victims. J Trauma Stress 13:89-100. doi:10.1023/A:1007725015225

33. Kulkarni M, Porter KE, Rauch SAM (2012) Anger, dissociation, and PTSD among male veterans entering into PTSD treatment. J Anxiety Disord 26:271-278. doi:10.1016/j.janxdis.2011.12.005

34. Kuwert P, Knaevelsrud C, Pietrzak RH (2014) Loneliness among older veterans in the United States: results from the National Health and Resilience in Veterans Study. Am J Geriatr Psychiatry 22(6):564-569. doi:10.1016/j.jagp.2013.02.013

35. Loos ME, Alexander PC (1997) Differential effects associated with self-reported histories of abuse and neglect in a college sample. J Interpers Violence 12(3):340-360. doi:10.1177/ 088626097012003002

36. Shevlin M, McElroy E, Murphy J (2015) Loneliness mediates the relationship between childhood trauma and adult psychopathology: evidence from the adult psychiatric morbidity survey. Soc Psychiatry Psychiatr Epidemiol 50:591-601. doi:10.1007/ s00127-014-0951-8

37. Leyro TM, Bernstein A, Vujanovic AA et al (2011) Distress tolerance scale: a confirmatory factor analysis among daily cigarette smokers. J Psychopathol Behav Assess 33(1):47-57. doi:10.1007/s10862-010-9197-2

38. Fetzner MG, Peluso DL, Asmundson GJG (2014) Tolerating distress after trauma: differential associations between distress tolerance and posttraumatic stress symptoms. J Psychopathol Behav Assess 36:475-484. doi:10.1007/s10862-014-9413-6

39. Marshall-Berenz EC, Vujanovic AA, Bonn-Miller MO et al (2010) Multimethod study of distress tolerance and PTSD symptom severity in a trauma-exposed community sample. J Trauma Stress 23(5):623-630. doi:10.1002/jts.2056

40. Dorahy MJ, van der Hart O (2015) DSM-5's posttraumatic stress disorder with dissociative symptoms: challenges and future directions. J Trauma Dissociation 16(1):7-28. doi:10.1080/ 15299732.2014.908806

41. Carlson EB, Waelde LC, Palmieri PA et al (2016) Development and validation of the Dissociative Symptoms Scale. Assess. doi:10.1177/1073191116645904

42. Weathers FW, Blake DD, Schnurr PP et al (2013) The life events checklist for DSM-5 (LEC-5). http://ptsd.va.gov

43. Weathers FW, Litz BT, Keane TM et al (2013) The PTSD checklist for DSM-5 (PCL-5). http://ptsd.va.gov

44. Hoge CW, Riviere LA, Wilk JE et al (2014) The prevalence of post-traumatic stress disorder (PTSD) in US combat soldiers: a head-to-head comparison of DSM-5 versus DSM-IV-TR symptom criteria with the PTSD checklist. Lancet Psychiatry 1:269-277. doi:10.1016/S2215-0366(14)70235-4

45. Lovibond SH, Lovibond PF (1995) Manual for the Depression Anxiety Stress scales, 2nd edn. Psychology Foundation, Sydney

46. Hawthorne G, Mouthaan J, Forbes D et al (2006) Response categories and anger measurement: do fewer categories result in poorer measurement? Development of the DAR5. Soc Psychiatry Psychiatr Epidemiol 41(2):164-172. doi:10.1007/s00127-005-0986-y

47. Russel DW (1996) UCLA Loneliness Scale (Version 3): reliability, validity, and factor structure. J Personal Assess 66(1):20-40. doi:10.1207/s15327752jpa6601_2

48. Simons JS, Gaher RM (2005) The Distress Tolerance Scale: development and Validation of a Self-Report Measure. Motiv Emot 29(2):83-102. doi:10.1007/s11031-005-7955-3

49. Muthén LK, Muthén BO (1998-2013) Mplus user's guide. Author, Los Angeles

50. Asparouhov T, Muthén BO (2014) Auxiliary variables in mixture modeling: A 3-step approach using Mplus. In: Mplus Web Notes (No. 15, Version 8). http://www.statmodel.com

51. Armour C, Karstoft KI, Richardson JD (2014) The co-occurrence of PTSD and dissociation: differentiating severe PTSD from dissociative-PTSD. Soc Psychiatry Psychiatr Epidemiol 49:1297-1306. doi:10.1007/s00127-014-0819-y

52. Stein DJ, Koenen KC, Friedman MJ et al (2013) Dissociation in posttraumatic stress disorder: evidence from the World Mental Health Surveys. Biol Psychiatry 73:302-312. doi:10.1016/j.biop sych.2012.08.022

53. Carlson EB, Dalenberg C, McDade-Montez E (2012) Dissociation in posttraumatic stress disorder Part I: definitions and review of research. Psychol Trauma Theory Res Pract Policy 4(5):479-489. doi:10.1037/a0027748

54. Lanius RA, Brand B, Vermetten E et al (2012) The dissociative subtype of posttraumatic stress disorder: rationale, clinical and neurobiological evidence, and implications. Depression Anxiety 29:701-708. doi:10.1002/da.21889

55. Forbes D, Lockwood E, Elhai JD et al (2015) An evaluation of the DSM-5 factor structure for posttraumatic stress disorder in survivors of traumatic injury. J Anxiety Disord 29:43-51. doi:10. 1016/j.janxdis.2014.11.004

56. Gentes EL, Dennis PA, Kimbrel NA et al (2014) DSM-5 posttraumatic stress disorder: factor structure and rates of diagnosis. J Psychiatr Res 59:60-67. doi:10.1016/j.jpsychires.2014.08.014 
57. Miller MW, Wolf EJ, Kilpatrick D et al (2013) The prevalence and latent structure of proposed DSM-5 posttraumatic stress disorder symptoms in U.S. national and veteran samples. Psychol Trauma Theory Res Pract Policy 5(6):501-512. doi:10.1037/ a0029730

58. Cardeña EB, Carlson EB (2011) Acute stress disorder revisited. Annu Rev Clin Psychol 7:245-267. doi:10.1146/annurev-clinpsy032210-104502

59. Bowins B (2004) Psychological defense mechanisms: a new perspective. Am J Psychoanal 64(1):1-26. doi:10.1023/B:TAJP. 0000017989.72521 .26

60. Blevins CA, Weathers FW, Davis MT et al (2015) The Posttraumatic Stress Disorder Checklist for DSM-5 (PCL-5): development and initial psychometric evaluation. J Trauma Stress 28(6):489-498. doi:10.1002/jts.22059

61. Bovin MJ, Marx BP, Weathers FW et al (2016) Psychometric properties of the PTSD Checklist for Diagnostic and Statistical
Manual of Mental Disorders-fifth edition (PCL-5) in veterans. Psychol Assess 28(11):1379-1391. doi:10.1037/pas0000254

62. Foa EB, Riggs DS, Massie ED et al (1995) The impact of fear activation and anger on the efficacy of exposure treatment for PTSD. Behav Ther 26(3):487-499. doi:10.1016/S00057894(05)80096-6

63. Forbes D, Parslow R, Creamer M et al (2008) Mechanisms of anger and treatment outcome in combat veterans with posttraumatic stress disorder. J Trauma Stress 21(2):142-149. doi:10. $1002 /$ jts. 20315

64. Jaycox LH, Foa EB (1996) Obstacles in implementing exposure therapy for PTSD: case discussions and practical solutions. Clin Psychol Psychother 3(3):176-184

65. Schauer M, Elbert T (2010) Dissociation following traumatic stress: etiology and treatment. J Psychol 218(2):109-127. doi:10. 1027/0044-3409/a000018 